\title{
POSSIBILITIES OF 3D PRINTER RAPIDSHAPE D30 FOR MANUFACTURING OF CUBIC SAMPLES
}

\author{
Tsanka Dikova ${ }^{1}$, Dzhendo Dzhendov ${ }^{2}$, Iveta Katreva ${ }^{2}$, Diana Pavlova ${ }^{3}$, Maksim Simov $^{3}$, \\ Svetlana Angelova ${ }^{3}$, Metodi Abadzhiev², Tsvetan Tonchev ${ }^{4}$ \\ ${ }^{1}$ Department of Medical and Biological Sciences, Faculty of Dental Medicine, \\ Medical University of Varna \\ ${ }^{2}$ Department of Prosthetic Dentistry and Orthodontics, Faculty of Dental Medicine, \\ Medical University of Varna \\ ${ }^{3}$ TRS Dental Technician, Medical College of Varna, Medical University of Varna \\ ${ }^{4}$ Department of Oral and Maxillofacial Surgery, Faculty of Dental Medicine, \\ Medical University of Varna
}

\section{ABSTRACT}

The purpose of the present paper is to establish experimentally the possibilities of the 3D printer Rapidshape D30 for the manufacturing of standard cubic samples from different types of monomers and to evaluate their geometrical accuracy and roughness.

Standard cubic samples with dimensions $5 \mathrm{~mm} \times 5 \mathrm{~mm} \times 5 \mathrm{~mm}$ were printed from all the materials, given by the producer, in two ways of dimensional orientation according to the base - horizontally and inclined at $45^{\circ}$. They were manufactured with 3D printer Rapidshape D30, working on a stereolithography principle with LED light.

It was established that:

* The samples, made of NextDent Surgical Guide, have the highest dimensional accuracy and the lowest deviation interval in both ways of placement. This polymer is characterized with the highest thickness of the polymerization layer $-0.100 \mathrm{~mm}$, but it is transparent, thus ensuring the entire polymerization throughout the depth of the whole layer. NextDent $\mathrm{C}+\mathrm{B}$, intended for manufacturing of temporary crowns and bridges, occupies a middle position in comparison to the other 6 materials.

* Nearly in all the materials the surface roughness of the cube with horizontal placement is lower than that of the cube printed inclined (average values of $\mathrm{Ra} 0.46-2.69 \mu \mathrm{m}$ and $\mathrm{Ra} 1.74-2.77$, respectively).

* In 3D printing the surface roughness depends on the thickness of the polymerization layer and the inclination to the printing direction. The thicker the polymerization layer and the larger the slope, the higher the surface roughness.

Keywords: 3D-printing, stereolithography, dimensional accuracy, surface roughness

Address for correspondence:

Assoc. Prof. Tsanka Dikova

Faculty of Dental medicine

Medical University of Varna

150, "Tzar Osvoboditel" str.

9000 Varna, Bulgaria

e-mail:tsanka_dikova@abv.bg

Received: March 11, 2016

Accepted: March 14, 2016

\section{INTRODUCTION}

Prosthetic constructions can be produced by five techniques: plastic deformation, additive and subtractive technologies, production of dense (compact) objects, as well as by the most modern approach for building up an object layer by layer. The group of subtractive technologies includes processes such as cutting, milling, filing, grinding, polishing and laser "milling". Additive technologies are stereolithog- 
raphy, fused deposition modeling, selective electron beam and laser melting, selective laser sintering and inkjet printing.

The new technologies show a rapid evolution during last 40 years due to the intensive development of new dental materials. The huge growth of computerization and digitalization has resulted in the creation of the Computer Aided Design (CAD) - Computer Aided Manufacturing (CAM) systems and their implementation in the fabrication of prosthetic restorations. The CAM unit may include different machines using various production processes - from milling to layer-upon-layer manufacturing via selective laser sintering/melting, stereolithography, etc.

The first CAD-CAM systems with an implementation in dental medicine are based on the subtractive technologies $(1,2,3,4)$. They are characterized by the following features:

* The dental construction is fabricated from a pre-shaped block;

* The material properties are always guaranteed;

* Processes of cutting or milling with mills or diamond disks in conventional milling machines with numerical control are applied;

* The path of the working instrument is carefully determined in order for the excess material to be removed;

* These technologies are implemented for the manufacturing of metal or ceramic crowns and bridges in the dental medicine.

The main advantage of these technologies is the production of complex shapes and objects in very short terms, which is impossible to obtain through the well known conventional production processes in the dental laboratory. On the other hand, all the CAD-CAM technologies still have some limitations concerning: 1) the precision fit of the inside contour of the restoration which depends on the size of the smallest usable tool; 2) the waste of a considerable amount of raw material - sometimes approximately 90 percent of the initial block (1); 3) abrasion wear of the milling tool and its short running cycles; 4) $\mathrm{mi}^{-}$ croscopic cracks on the ceramic surfaces due to the brittle nature of this material (2).

The aforementioned drawbacks can be overcome by using additive technologies for manufacturing objects through building them up layer upon lay- er, i.e. Additive Manufacturing (AM). The American Society for Testing and Materials (ASTM) defines the term "additive manufacturing” as: „the process of joining materials to make objects from 3D model data, usually layer upon layer, as opposed to subtractive manufacturing methodologies" $(1,5)$.

Stereolithography, Fused Deposition Modeling (FDM), Selective Electron Beam Melting (SEBM), Selective Laser Sintering (SLS)/Selective Laser Melting (SLM) and Inkjet Printing (IP) are currently the most frequently applied methods in the dental field $(1,2,4,6)$.

Stereolithography is implemented in the fabrication of physical models of human anatomy, surgical guides in implantology, surgical treatment planning, constructing of custom implants, temporary crowns and bridges, molds for metal casting. Fused deposition modeling is applied for developing wax patterns for metal casting. SEBM allows the manufacturing of custom implants and prostheses for maxillofacial orthopedics and surgery from pure $\mathrm{Ti}$, Ti-6Al-4V and Co-Cr alloys. It gives the possibility of producing objects with porous structure but the accuracy of the details is low: $0.3-0.4 \mathrm{~mm}$, and the surface roughness is pretty high $-R_{a}$ up to $25 \mu \mathrm{m}$. For this reason SEBM is not suitable for the manufacturing of denture frameworks, crowns and bridges. Selective Laser Sintering (SLS) is a term used when working with polymers and ceramics, and Selective Laser Melting (SLM) is connected with metal and alloy treatment. This methodology is very appropriate for implementation in dental medicine and especially the prosthetic dentistry for its potential to work with the whole variety of dental materials - thermoplastic polymers, modeling wax, metals and alloys ( $\mathrm{Ti}$ and its alloys, Co-Cr alloys, stainless steel), ceramics and thermoplastic composites. It is used for the production of facial prosthesis; functional frames and custom molds in the field of tissue engineering from composites; orthopedic and dental implants (with porous structure); crowns, bridges and metal frameworks of cast dentures in prosthodontics. The technology of inkjet printing produces dental models, guides for braces in orthodontics, surgical guides for implant placement and different types of intraoral splints and appliances for sleep apnea treatment. 
The working process of the Rapidshape D30 3D printer is based upon stereolithography. The objects are obtained through layered manufacturing by light curing of a liquid monomer. LED lighting is used as a light source. This $3 \mathrm{D}$ printer is the newest generation and assures the manufacturing of highly precise and accurate constructions (with an accuracy of $\pm 29 \mu \mathrm{m}$ ) (7). It is possible to print models, customized trays, surgical guides, temporary crowns and bridges, casting patterns, denture bases, gingival masks and protection appliances. Different materials with variable transparency and color are applied for each of the in- rials and to estimate their dimensional accuracy and roughness.

\section{EXPERIMENTAL METHODS}

Standard samples have been prepared - 10 cubes with dimensions $5 \mathrm{~mm} \times 5 \mathrm{~mm}$ x $5 \mathrm{~mm}$ from each of the available materials (Table 1 and Fig. 1). The samples have been printed simultaneously in two ways of dimensional orientation. The first position of the sample is with its edge parallel to the base and its diagonal plane perpendicular to the base. In the second way of orientation of the cube its wall is

Table 1. Type of material and thickness of the layer for printing [mm], defined by the software

\begin{tabular}{lc|c|cccc}
$\begin{array}{l}\text { Material } \rightarrow \\
\text { Orientation of the } \\
\text { sample } \downarrow\end{array}$ & $\begin{array}{c}\text { NextDent } \\
\text { Model }\end{array}$ & $\begin{array}{c}\text { NextDent } \\
\text { Model Ortho }\end{array}$ & $\begin{array}{c}\text { NextDent } \\
\text { Surgical } \\
\text { Guide }\end{array}$ & $\begin{array}{c}\text { NextDent } \\
\text { C+B }\end{array}$ & $\begin{array}{c}\text { NextDent } \\
\text { Tray }\end{array}$ & $\begin{array}{c}\text { NextDent } \\
\text { Base }\end{array}$ \\
$\begin{array}{l}\text { Horizontal } \\
\text { position }\end{array}$ & 0,035 & 0,050 & 0,100 & 0,050 & 0,050 & 0,035 \\
$\begin{array}{l}\text { Tilted position } \\
\text { at } 45^{\circ}\end{array}$ & 0,035 & 0,050 & 0,100 & 0,050 & 0,050 & 0,035 \\
$\begin{array}{l}\text { Final light } \\
\text { curing, [min] }\end{array}$ & 10 & 10 & 10 & 30 & 10 & 10 \\
\hline
\end{tabular}

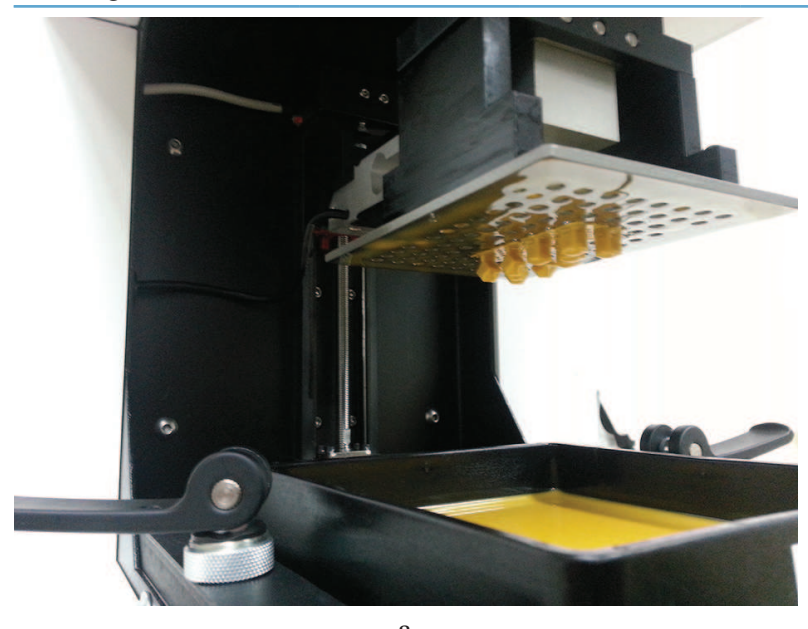

a

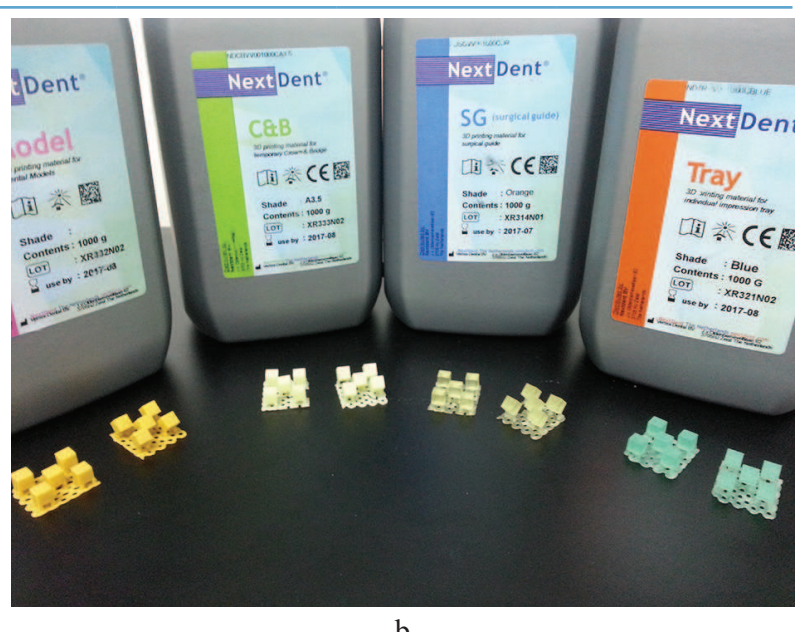

b

Fig. 1. Process of the samples manufacturing - a) and as-received samples of different resins' types - $b$ )

dications listed above. The information about accuracy and surface roughness of the objects, printed by Rapidshape D30 is still insufficient since the machine is the newest generation.

The purpose of the present article is to establish experimentally the potential of the $3 \mathrm{D}$ printer for the fabrication of standard samples of the assorted mate-
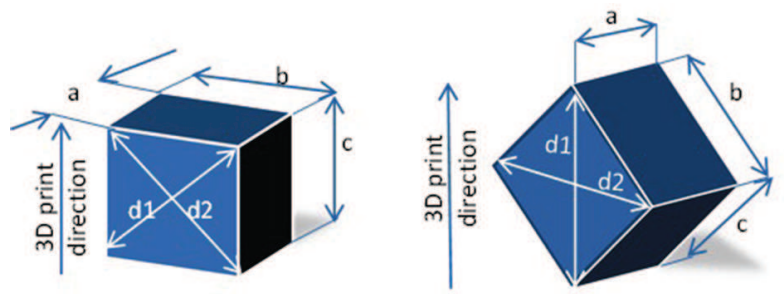

Fig. 2. Scheme of measuring of the $3 D$ printed samples 


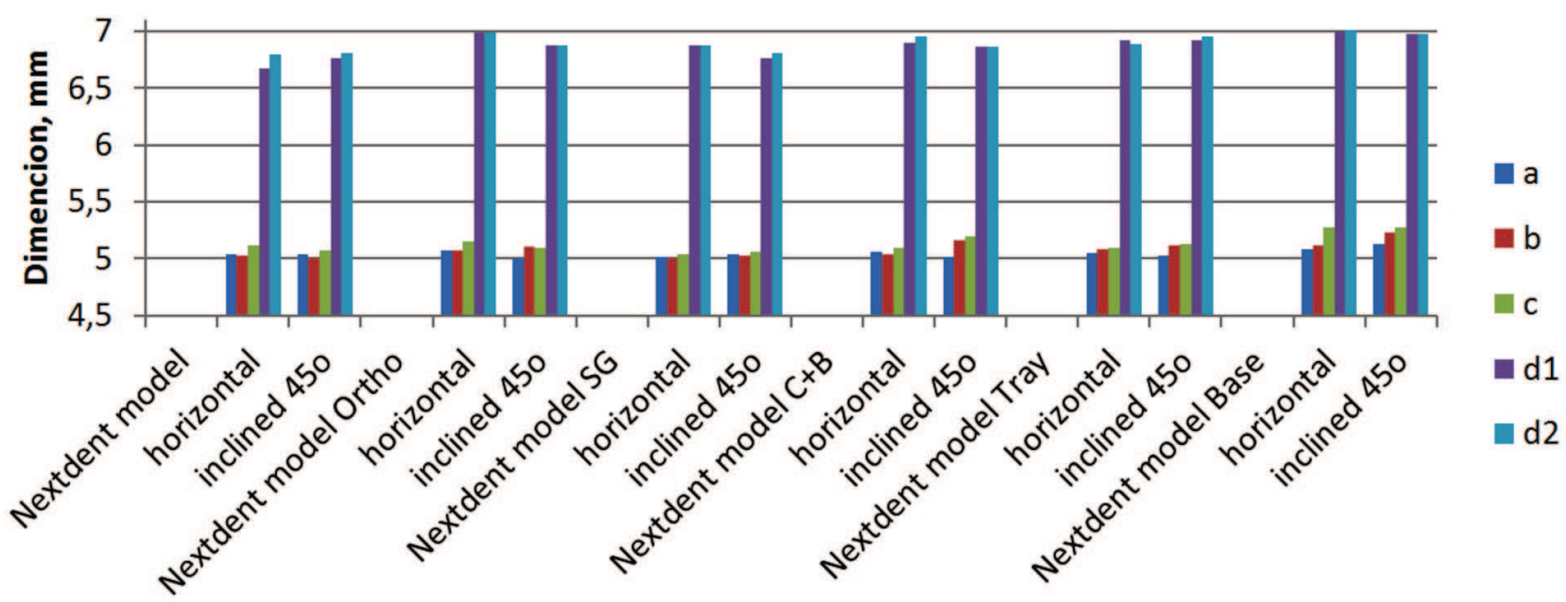

Fig. 3. Dimensions of cubic samples, manufactured of different materials by 3D printer Rapidshape D30

parallel to the base. All the samples underwent final light curing after the process of printing has been completed. Their geometrical accuracy has been studied then by a measuring of the dimensions of the walls and diagonals (Fig. 2) of 3 samples of each material and each way of dimensional position. The surface roughness of 3 of the cubes' walls, which are differently situated according to the printing direction, was inspected through a measurement of the average deviation of the roughness $R a$ by the profilometer Taylor Hobson Surtronik 3.

\section{RESULTS AND ANALYSIS}

\section{Accuracy of the Dimensions}

The dimensions of the cubic samples, printed by 3D printer Rapidshape D30 are shown on Fig. 3. The experimental objects, manufactured from NextDent

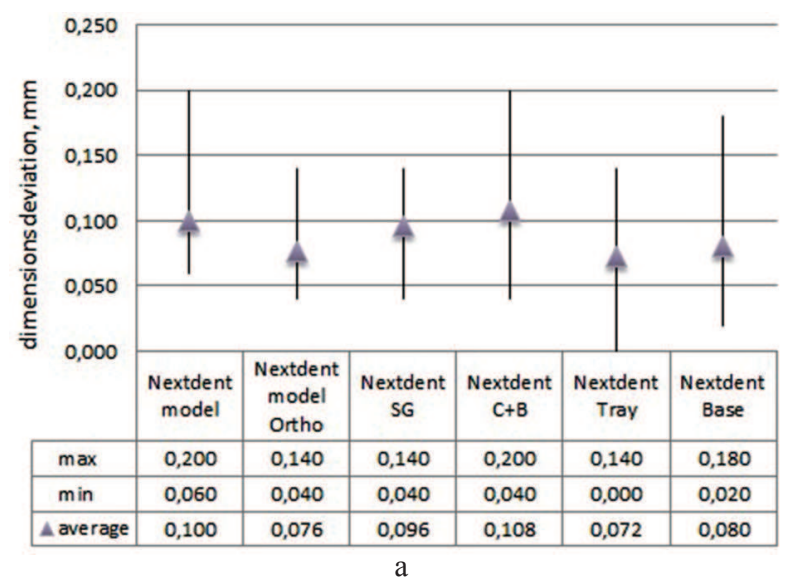

Surgical Guide, show the highest accuracy no matter of the direction of placement during printing. The accuracy is decreasing in horizontally printed samples made of NextDent Tray and NextDent C+B. The diagonals of the cubes, printed from NextDent Surgical Guide show least difference also.

The values of the maximum deviation of the dimensions of the cubic samples, printed in horizontal position, as well as inclined at $45^{\circ}$, are presented on Fig. 4. These results verify the data from Fig. 3. It is obvious that the smallest range of variation and the smallest average deviation are those of the specimens from NextDent Surgical Guide $(0.100 \mathrm{~mm}$ and 0.096 $\mathrm{mm})$, NextDent Model Ortho $(0.100 \mathrm{~mm}$ and 0.076 $\mathrm{mm})$ and NextDent Tray (0.140 $\mathrm{mm}$ and $0.072 \mathrm{~mm})$ in horizontal position (Fig. 4-a). Almost the same tendency is found among the inclined samples (Fig.4-

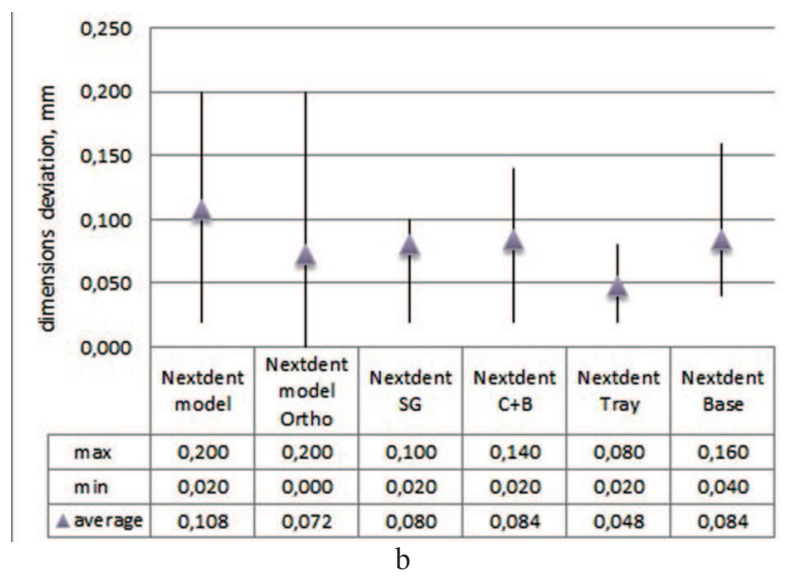

Fig. 4. Maximum deviation of the dimensions of cubic samples printed in horizontal position - a) and inclined at $45^{\circ}$ $-b)$ 
Tsanka Dikova, Dzhendo Dzhendov, Iveta Katreva et al.

b). The smallest range of variation of the dimensions is observed in the experimental objects from NextDent Tray, NextDent Surgical Guide and NextDent C+B and it is as follows: $0.060 \mathrm{~mm}, 0.080 \mathrm{~mm}$ and 0.120 $\mathrm{mm}$. The NextDent $\mathrm{C}+\mathrm{B}$ resin for temporary crowns and bridges occupies a middle position according to its dimensional accuracy in comparison to the other 6 types of resin materials.

The analysis of the results reveals that the highest dimensional accuracy and the least range of variation is presented by the samples, printed with the greatest thickness of the layer $-0.100 \mathrm{~mm}$ for NextDent Surgical Guide and $0.050 \mathrm{~mm}$ for NextDent Model Ortho, NextDent Tray and NextDent C+B. Further consideration of the data determines that the thickness of the polymerization layer, given by the producer, depends on the optical properties of the resin material. For that reason the opaque resins with dense color (NextDent Base, NextDent C+B, NextDent Model) are applied for polymerization in a thinner layer, and the transparent ones (NextDent

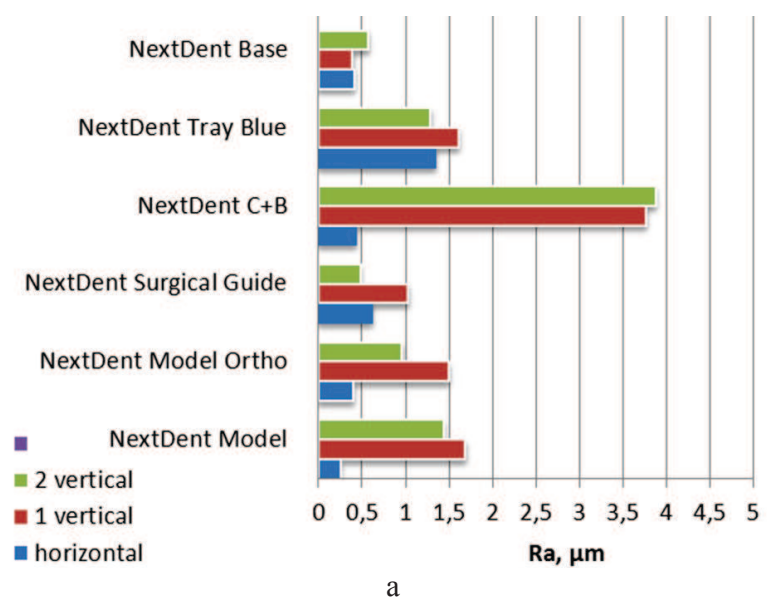

Surgical Guide and NextDent Tray) - in a layer with a greater thickness. The polymerization process takes place almost throughout the entire layer of the transparent resins although its thickness is higher, compared to the opaque resin materials. Thus, only a small part of the layer is left for the final polymerization which reduces the internal tensions and increases the accuracy of the object.

\section{Surface Roughness}

The samples, placed horizontally, have much lower roughness of the walls as compared with those printed in an inclined position (Fig. 5). The lowest roughness is observed in the walls positioned horizontally relative to the base. Their arithmetic mean deviation $R a$ of the roughness is within the range $0.5 \mu \mathrm{m}$ and even less for almost all types of resins. As the 3D printer works on the principle of curing with LED lighting, not laser, no difference was expected between the roughness of the horizontal and the vertical walls. However, the data

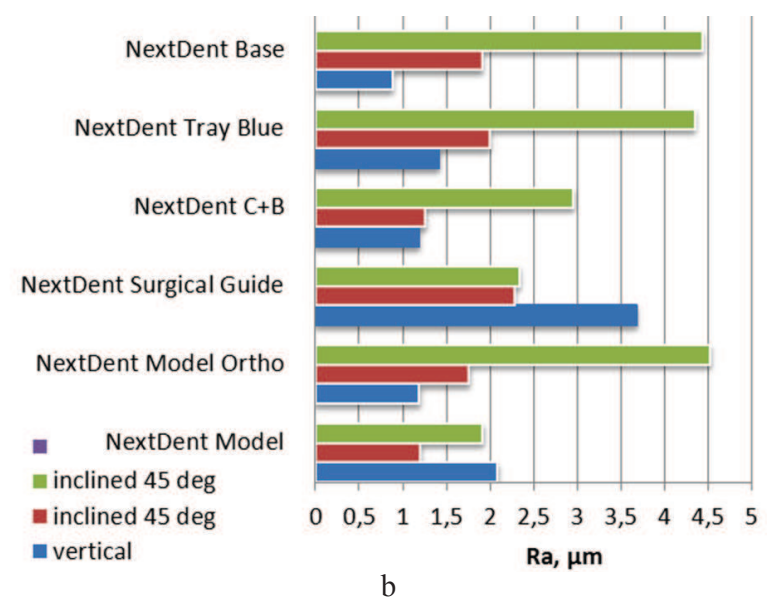

Fig. 5. The arithmetic mean deviation $R$ a of the roughness of surfaces of cube $3 D$ printed from different resins in a horizontal position - a) and inclined at $45^{\circ}-b$ )

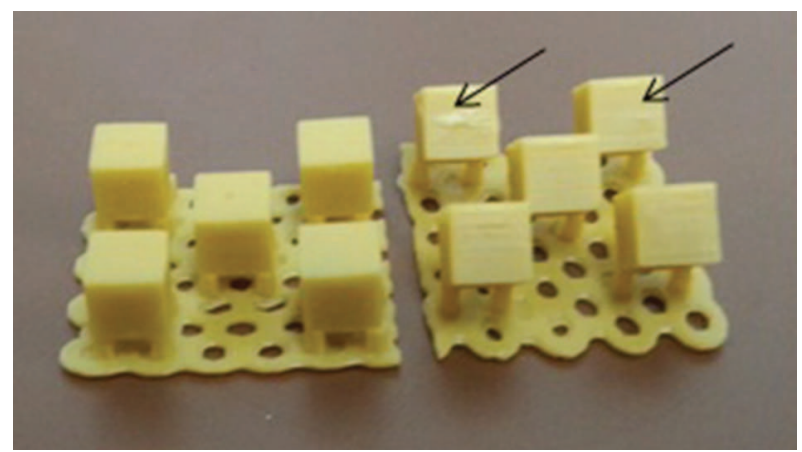

Fig. 6. Samples made of NextDent Model from the measurements shows almost 3 times greater roughness of the vertical walls (Fig. 5-a). Similar values of $R a$ are obtained for the vertical walls of cubes, printed in an inclined position. The lowest $R a$ of the vertical walls among horizontally positioned samples has the resins NextDent Base, NextDent Surgical Guide and NextDent Model Ortho. Among the inclined experimental objects the minimum value of $R a$ of the vertical walls is found in NextDent Base, 
Possibilities of 3D Printer Rapidshape D30 for Manufacturing of Cubic Samples

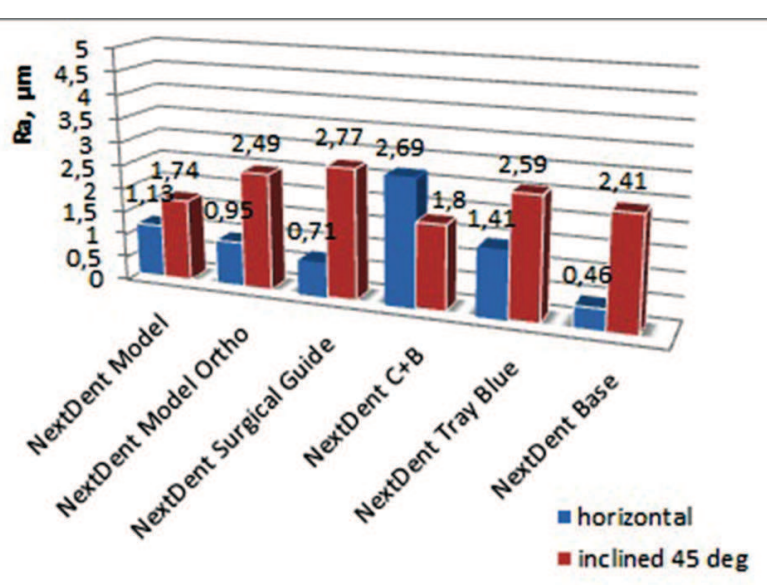

Fig. 7. Average values of $R$ a of cube $3 D$ printed from different resins

NextDent Model Ortho and NextDent C+B. In other words, the vertical walls with the least roughness are those of the samples, printed from resins with a thinner layer of polymerization $(0.035-0.050 \mathrm{~mm})$. Therefore, the most likely roughness of the vertical walls is caused by instability and uncertainties in the table movement in the direction of print.

The walls of the experimental specimens in inclined position at $45^{\circ}$ relative to the base are with the greatest roughness (Fig. 5-b). The values of $\mathrm{Ra}$ of the walls that are closer to the support are smaller, i.e. the walls which are printed at the beginning of the process. The values of roughness are of the greatest range for the walls that are printed at the end of the polymerization process (Fig. 6). They reach $1.92 \mu \mathrm{m}$ in NextDent Model and are up to $4.53 \mu \mathrm{m}$ in NextDent Model Ortho. Upon surveillance the different layers and the surface defects, marked by arrows in Fig. 6 are clearly visible. The surface defects are more likely to be caused by poor cleaning of the samples from the liquid monomer and its subsequent polymerization during the final curing. The traces of the individual layers are directly dependent on their thickness, which is confirmed by the data on the average Ra values (Fig. 7). The greatest value is found in the objects from NextDent Surgical Guide, printed inclined at $45^{\circ}$ with thickness of the layer $0.100 \mathrm{~mm}$ and it is the maximum value found among all the resins tested in the study. Hence, the roughness of the samples is directly dependent on the thickness of the polymerization layer and the inclination of the surface to the base, respective to the direction of print. The thicker polymerization layer and the larger inclination to the direction of print, the greater the roughness is.

\section{CONCLUSIONS}

The study of the geometrical accuracy and the roughness of the cubic samples, printed in two ways of placement - horizontal and inclined at $45^{\circ}$ by $3 \mathrm{D}$ printer Rapidshape D30, working at the principle of stereolithography with LED lighting, showed that:

* The highest dimensional accuracy and the least range of deviation in both positions of print have the samples, made of the resin NextDent Surgical Guide. The material has the greatest thickness of the polymerization layer $-0.100 \mathrm{~mm}$, but it is transparent and provides entire polymerization throughout its mass. In regard to dimensional accuracy and range of deviation the resin for temporary crowns and bridges NextDent $\mathrm{C}+\mathrm{B}$ takes medial position compared to the other 6 types.

* The roughness of the surfaces of the cubes with horizontal position of print is less than those of the cubes, printed inclined in almost all types of resins (mean values of $\mathrm{Ra} 0.46-2.69 \mu \mathrm{m}$ and $\mathrm{Ra}$ $1.74-2.77 \mu \mathrm{m}$, respectively).

* In the process of 3D printing of objects, the roughness of the surface depends on the thickness of the layer which undergoes polymerization and the inclination to the direction of the print. The higher thickness of polymerization layer and inclination, the greater roughness.

\section{ACKNOWLEDGEMENTS}

The present study is supported by the project with contract 502/19, 12 Dec 2014, of the Fund for Scientific Investigations, Ministry of Education and Science of Bulgaria.

\section{REFERENCES}

1. van Noort R., The future of dental devices is digital. Dental Materials 2012;28:3-12.

2. Torabi K., Farjood E., Hamedani Sh. Rapid Prototyping Technologies and their Applications in Prosthodontics, a Review of Literature. J Dent Shiraz Univ Med Sci., March 2015; 16(1): 1-9. 
3. Witkowski S. (CAD-)/CAM in der Zahntechnik: Buyer's Guide 2003. Zahntech Mag 2002; 6: 696-709.

4. Dikova T, Dzhendov D, Simov M, KatrevaBozukova I, Angelova S, Pavlova D, Abadzhiev $\mathrm{M}$, Tonchev T. MODERN TRENDS IN THE DEVELOPMENT OF THE TECHNOLOGIES FOR PRODUCTION OF DENTAL CONSTRUCTIONS. J of IMAB. 2015 OctDec;21(4):974-981. doi: http://dx.doi.org/10.5272/ jimab.2015214.974.

5. Dovbish VM, Zabednov PV, Zlenko MA. Additivnie tehnologii I izdelia iz metala, 57p. (in Russian).

6. Dikova Ts., N. Panova, M. Simov. Application of Laser Technologies in Dental Prosthetics, Int. Journal "Machines, Technologies, Materials" 2011;6:32-35, http://mech-ing.com/journal/6-2011. html.

7. The innovative solution for efficiency and precision: Our new 3D-Printer for dental application. Available from http://www.rapidshape. de/portfolio/d30, (22 Jan 2016). 